\title{
Investigation of the relationship between schizophrenia and toxoplasmosis in Van province, Turkey
}

\begin{tabular}{|c|c|}
\hline \multirow[t]{2}{*}{$\begin{array}{l}\text { Original } \\
\text { Article }\end{array}$} & $\begin{array}{l}\text { Abdurrahman Ekici', Damla K Timuçin², Esra Gürbüz }{ }^{3} \text {, Ahmet H Ünlü }{ }^{4} \text {, Selahattin } \\
\text { Aydemir }{ }^{1} \text {, Hasan Yilmaz }\end{array}$ \\
\hline & $\begin{array}{l}\text { Departments of Parasitology, Van Yüzüncü Yll University, Faculty of Medicine }{ }^{1} \text {, SBÜ Van } \\
\text { Training and Research Hospital for Mental and Neurological Diseases }{ }^{2} \text {, and Infectious } \\
\text { Diseases and Clinical Microbiology }{ }^{3} \text {, Van Yüzüncü Yll University, Gevaş Vocational School }{ }^{4} \text {, } \\
\text { Van, Turkey }\end{array}$ \\
\hline
\end{tabular}

\begin{abstract}
Background: Schizophrenia is a worldwide, serious neuropsychiatric disorder of unknown cause. Previous studies showed that infectious agents may play a role in its etiology. Among them, T. gondii was strongly hypothesized.

Objectives: The aim of this study was to determine the IgG and IgM seroprevalence of T. gondii in schizophrenia patients.

Subjects and Methods: The study was conducted on 190 patients diagnosed with schizophrenia in Van province, Turkey. Also included as the control group were 100 healthy individuals. Anti-T. gondii IgG and IgM antibody positivity was determined by enzyme-linked immunosorbent assay (ELISA) in blood samples taken from both groups.

Results: Anti-T. gondii IgG antibody was detected in 120 schizophrenic patients (63.2\%), and in 100 (29\%) healthy controls. Anti-T. gondii IgM antibody seropositivity was not detected in either group.

Conclusion: In line with the data obtained in the study carried out in Van province, it was concluded that individuals infected with T. gondii were more likely to develop schizophrenia. Further studies are recommended to prove the relationship between T. gondii and schizophrenia.
\end{abstract}

Keywords: Schizophrenia, toxoplasmosis, Toxoplasma gondii, Van.

Received: 15 November, 2020, Accepted: 2 February, 2021.

Corresponding Author: Abdurrahman Ekici, Tel.: +905 077042400, E-mail: abdurrahman2400@gmail.com

Print ISSN: 1687-7942, Online ISSN: 2090-2646, Vol. 14, No. 1, April, 2021.

\section{INTRODUCTION}

The only species of Toxoplasma in the Apicomplexa group that can parasitize humans, other mammals and poultry is T. gondii. It was first described by Charles Nicole and Lovis Monceaux at the Pasteur Institute in Tunisia in 1908 by isolation from Ctenodactylus gundi, a North African rodent. The asexual and sexual life cycle of Toxoplasma was first established simultaneously by two research groups in $1970^{[1,2]}$.

Toxoplasmosis is a systemic infection caused by $T$. gondii affecting mammals and avian species worldwide. Cats and other Felidae species are both intermediate, and final hosts of T. gondii. Intermediate hosts comprise many mammals including humans, and poultry ${ }^{[3]}$. It has serious clinical complications on human health and affects approximately 30\%-60\% of the population in both developed and developing countries $^{[4]}$. The prevalence of $T$. gondii varies according to the lifestyle, socioeconomic status, and eating habits of the population in different geographical regions of the world ${ }^{[5]}$. The primary route of transmission of the parasite is through contact with the feces of infected felines, particularly domestic cats. Additionally, T. gondii can be transmitted through ingestion of improperly cooked infected meat, transplacentaly from mother-to-fetus, and from soil or water contaminated with oocysts ${ }^{[6]}$. When T. gondii infects pregnant women, it can cause a congenital syndrome that includes deafness, retinal damage, seizures, and mental retardation. It may cause severe central nervous system symptoms in immunocompromised individuals ${ }^{[7]}$.

There are 2 forms of toxoplasmosis in humans. The first is caused by actively reproducing tachyzoite forms in the early acute phase of infection. In the second chronic or latent form, bradyzoites or tissue cysts are found in the muscles and brain ${ }^{[8]}$. Due to the high neurotropic effect of T. gondii, the most affected tissue in the body in the chronic process is the brain ${ }^{[4]}$. Toxoplasmosis is usually asymptomatic, but in immunocompromised patients, it can lead to severe clinical complications, such as retinochoroiditis, myocarditis, and meningoencephalitis, which can lead to death ${ }^{[6]}$.

ELISA is widely used in the diagnosis of toxoplasmosis, especially in the search for IgG antibodies. However, an IgG titer alone, no matter the level, can not predict whether the infection occured in the recent or distant past but may be considered as ameasure of exposure to the infection. Avidity testing 
is required to make this distinction. Although IgM antibodies are detected earlier than IgG antibodies and decrease faster they are not an absolute marker of acute infection because they could persist for years after acute infection. Therefore, persisting IgM antibodies have no clinical significance ${ }^{[9]}$.

Schizophrenia, usually starts in youthful individuals as a mental disorder with symptoms. The course and end may differ between individuals, resulting in different presentations that may be positive (hallucinations, illusions, thought and movement disorders) or negative (lack of emotional response, lack of excitement, anhedonia, no social interaction, planned activities, difficulty of maintaining chores) ${ }^{[10]}$. Schizophrenia with negative, positive, and cognitive symptoms is one of the most common and wellknown psychotic disorders ${ }^{[11]}$, affecting $1 \%$ of the world population $^{[12]}$. According to the 2016 data of the World Health Organization, schizophrenic patients of every type and in every socioeconomic class comprise more than 21 million worldwide, while in Turkey it has reached approximately 500,000 ${ }^{[11]}$. Although many factors are known to play a role in its etiology, the interaction between genetic predisposition and environmental stress is important. Interestingly, numerous epidemiological studies have shown that microorganisms may have a possible role in the etiopathogenesis of schizophrenia cases. As a result of the insufficiency of genetic studies on schizophrenia, the interest of researchers has focused on infectious risk factors. In many studies, the relationship between infectious agents and schizophrenia has been examined and its place in schizophrenia etiology has been discussed ${ }^{[4,13-15]}$.

The aim of this study was to determine the seroprevalence of $T$. gondii in patients with schizophrenia and reveal the importance of toxoplasmosis as a risk factor in these patients.

\section{SUBJECTS AND METHODS}

This cross-sectional study was conducted on 190 patients diagnosed with schizophrenia for the first time and followed-up at the Community Mental Health
Center between 20 December 2018 and 1 October 2020 in Van province, Turkey. Also included as the control group were 100 healthy individuals without schizophrenia. T. gondii IgG and IgM antibodies were investigated by ELISA method for the determination of chronic or latent toxoplasmosis, and for the detection of active infection, respectively.

Serum was separated from the blood samples of the patients and stored in a deep freezer at $-80^{\circ} \mathrm{C}$. Anti- $T$. gondii immunoglobulins $\mathrm{G}$ and $\mathrm{M}$ antibody levels (AL) in blood samples were determined using an enzymelinked immunosorbent assay (ELISA) (Vircell SL, Granada, Spain). The serum samples were allowed to thaw at room temperature before testing in accordance with the manufacturer's instructions. An $\mathrm{AL}<9$ was evaluated as negative, those between 9 and 11 as borderline, and $>11$ as positive $(\mathrm{AL}=$ [optical density $(O D) /$ mean OD of cut off serum] $\times 10$ ).

Statistical analysis: Minitab 14 package program was used for all statistical analysis. Z test and Fisher's exact tests were used to determine statistical significance and $P<0.05$ was considered significant.

Ethical considerations: Approval of the ethics committee with decision number 11 was obtained on 21.12.2018 from the Van Yuzuncu Yil University NonInvasive Ethics Committee. Informed consents were obtained from patients and controls

\section{RESULTS}

In this study, T. gondii IgG antibody was detected in $120(63.2 \%)$ of the 190 schizophrenia patients, comprising 75/120 males (62.5\%) and 45/70 females (64.3\%). In the control group, anti-T.gondii IgG antibody was detected in 29 of 100 healthy controls (29\%), comprising 15/44 males (34.1\%) and 14/56 females (25\%). Anti-T. gondii IgM antibody seropositivity was not detected in either group. In the patient and control groups, there were no statistically significant differences between, gender, and T. gondii positivity. However, a statistically significant relationship was found between schizophrenia and Toxoplasma IgG antibody positivity $(P=0.001)$ (Table 1$)$.

Table 1. Comparative distribution of the prevalence of anti-T. gondii IgG antibody by gender and group.

\begin{tabular}{|c|c|c|c|c|c|}
\hline \multirow{2}{*}{ Groups } & \multirow{2}{*}{ Gender } & \multicolumn{2}{|c|}{ Positive } & \multirow{2}{*}{\multicolumn{2}{|c|}{$\begin{array}{c}\text { Statistical analysis } \\
P \text { values }\end{array}$}} \\
\hline & & No. & $\%$ & & \\
\hline \multirow{3}{*}{ Patient } & Female (n: 70) & 45 & 64.3 & \multirow{3}{*}{$=0.8^{*}$} & \multirow{6}{*}{$<0.001^{* *}$} \\
\hline & Male (n: 120) & 75 & 62.5 & & \\
\hline & Total & 120 & 63.2 & & \\
\hline \multirow{3}{*}{ Control } & Female (n: 56) & 14 & 25.0 & \multirow{3}{*}{$=0.8^{*}$} & \\
\hline & Male (n: 44) & 15 & 34.1 & & \\
\hline & Total & 29 & 29.0 & & \\
\hline
\end{tabular}




DISCUSSION

The importance of toxoplasmosis in public health has beenignoredformanyyears sinceitislargelyasymptomatic. T. gondii has been mainly investigated in pregnant women and immunosuppressed patients. In recent years, parallel to technological developments in the field of health, it has been noted that infection with T. gondii may have different results. It is also clear that this parasite, which resides in brain tissue, has been associated with many outcomes that can change the behavior of humans and some animals $s^{[9]}$. The investigation of the potential effects of the infection on human behavior was based on behavioral manipulative studies of T. gondii among infected mice and cats. In these studies, the parasite induced behavioral changes in the host. It was observed that Toxoplasma impaired learning and memory in mice and caused changes in the behavior of both mice and rats. Due to these behavioral changes, it was noted that the rats became easy prey for hunting cats and thus, the parasite could continue to complete its life cycle ${ }^{[4]}$.

Researchers found that mice infected with T. gondii had decreased instinctive fear of cat urine and impaired memory functions ${ }^{[16]}$. These studies with rodents led to the idea that the neurocognitive changes caused by the parasite may not be specific to mice alone, and that T. gondii may cause potential behavioral and/or neuropsychiatric disorders in humans. The relationship between T. gondii and behavioral states, such as psychiatric disorder, impulsivness, and impaired neurocognitive processes, has been investigated in humans, particularly in schizophrenics ${ }^{[17]}$.

Some cases of acute toxoplasmosis in adults were associated with psychiatric symptoms such as hallucinations and delusions ${ }^{[18,19]}$. Two separate studies, considered that individuals who somehow had close relationships with cats during childhood, constituted a risk factor for schizophrenia and bipolar disorder diseases $^{[20,21]}$. Although these findings may not be related directly to toxoplasmosis, they may be important indications that infectious agents play a role in the etiology of these diseases.

The following information was obtained from investigations of the relationship between schizophrenia and toxoplasmosis in different countries. A study conducted by Esquivel et al. ${ }^{[22]}$ in Mexico, reported 20\% seroprevalence of $T$. gondii in schizophrenia patients, while it was $5.3 \%$ in the healthy individuals. Alipour et $a l_{.}^{[23]}$, estimated $T$. gondii seroprevalence in Iran as $67.7 \%$ in the schizophrenia patients, and $37.1 \%$ in the healthy individuals. Emelia et al. ${ }^{[24]}$ reported in Malaysia, a seroprevalence of $T$. gondii in $61.1 \%$ schizophrenia patients, and $40.8 \%$ in the healthy individuals, and the seropositivity rate of anti-T. gondii IgG antibody in the schizophrenia patients was significantly related. In a study conducted by Khademvatan et al. ${ }^{[25]}$ in Iran, the seroprevalence of $T$. gondii was reported to be higher in the schizophrenic women when compared to the healthy women and men. In their study conducted in the Netherlands, Ladee et al. ${ }^{[26]}$ drew attention to the presence of uncommon schizophreniform features in those with chronic toxoplasmosis, acquired in childhood or early adulthood. The researchers noted that in some instances, a neurasthenic prodromal phase was followed by suspicious paranoia or paranoid delusions.

From Istanbul in Turkey, Yüksel et al. ${ }^{[27]}$ recorded a seroprevalence of $T$. gondii of $60.7 \%$ in the schizophrenia patients, and $45.3 \%$ in healthy blood donors; while in Elazığ, Çetinkaya et al.$^{[8]}$ reported that the seroprevalence of $T$. gondii was $66 \%$ in the schizophrenia patients, and $22 \%$ in the healthy individuals. Cevizci et al. ${ }^{[28]}$ in a similar study, reported that the seroprevalence of T. gondii was $33.3 \%$ in the schizophrenic patients, and $21.7 \%$ in the healthy individuals. In another study by Tamer et al. ${ }^{[29]}$, the seroprevalence of $T$. gondii was reported to be $40 \%$ in schizophrenia patients, as compared to $13.5 \%$ in the healthy individuals.

Tanyuksel et $a l^{[30]}$ studied first-episode schizophrenia patients, and reported anti-T. gondii IgG positivity of $43.8 \%$ in the patients, while it was $32.5 \%$ in the healthy individuals. Also, Dogruman et $a l^{[31]}$ reported a prevalence rate of $47.7 \%$ in the schizophrenia patients, and $21.6 \%$ in the group of healthy individuals. A study conducted in the USA ${ }^{[32]}$ examining 257 individuals with T. gondii antibodies, revealed 99 (38.5\%) attempted suicides with high antibodies titers, and 119 (46.3\%) had recurrent mood disorders. To investigate the relationship between toxoplasmosis and suicide attempts in Turkey, a conducted study, reported T. gondii positivity of $41 \%$ in the individuals who attempted suicide, versus $28 \%$ in the controls. This significant recorded difference showed that there may be a causal relationship between T. gondii positivity and the etiology of suicide attempts $^{[33]}$. A meta-analysis of 42 studies conducted by Torrey et al. ${ }^{[34]}$ covering the period from 1953-2007 revealed that those diagnosed with schizophrenia were nearly 3 times as likely to carry T. gondii. Additionally in a study conducted by Mortensen et $a l^{\left[{ }^{[35]}\right.}$ in Denmark, in which they matched the sera collected from 71 individuals with schizophrenia before the age of 18 with appropriate controls, T. gondii IgG antibodies were significantly higher than in the controls.

Our study is the first to investigate the relationship between schizophrenia and toxoplasmosis in the Van province. We found that $63.2 \%$ of 190 schizophrenia patients had anti-T. gondii IgG antibodies indicating previous exposure to infection. When the patients diagnosed with schizophrenia were compared with the control group, toxoplasmosis was observed at a higher rate and this difference was found to be statistically significant $(P<0.005)$. 
Conclusion: The data obtained in the current study, confirms that individual infection with T. gondii would likely lead to development of schizophrenia, and that toxoplasmosis was a causal contributor to schizophrenia. In addition, it was concluded that apparently healthy individuals with T. gondii positivity should be evaluated in terms of schizophrenia for early diagnosis and should be included in the possible risk group for schizophrenia. In order to reveal the relationship between schizophrenia and toxoplasmosis more precisely, it is necessary to conduct comprehensive studies on the interactions of the parasite with the human brain. It is believed that determining the role of $T$. gondii in schizophrenia will pave the way for new treatments, prevention, and control methods.

Authors`contributions: Ekici A, Yılmaz H and Ünlü AH conceived the study, and wrote the manuscript. Timuçin DK and Gürbüz E shared in the study design. Aydemir $S$ performed the experiments and analyzed the data. All authors revised the manuscript.

Competing interest: The authors declare that there are no competing interests.

Funding statement: The research was not funded by any institution.

\section{REFERENCES}

1. Hutchison W, Dunachie J, Siim JC, Work K. Coccidian-like nature of Toxoplasma gondii. Br Med J 1970; 1(5689): 142-144.

2. Frenkel J, Dubey J, Miller NL. Toxoplasma gondii in cats: fecal stages identified as coccidian oocysts. Science 1970; 167(3919):893-896.

3. Pekmezci D, Pekmezci GZ. Can toxoplasmosis make behavioural alterations in cats? Etlik Vet Mikrobiyol Derg 2016; 27(2):149-154.

4. Karabulut N. Toxoplasma and Schizophrenia. Türk Mikrobiyol Cem Derg 2013; 43(2):39-44.

5. Doğan N, Akdas I, Essizoglu A, Güleç G. Serological and molecular investigation of the presence and association of Toxoplasma gondii in bipolar affective disorder and schizophrenic patients. Flora 2018; 23(3):142-149

6. Akgül Ö. The effects of latent Toxoplasma gondii infection on the behavior and personality characteristics of university students. Alpa Psychiatry 2020; 21:70-76.

7. Yüksel P, Kocazeybek B. Do microorganisms have a role in neuropsychiatric diseases? Flora 2013; 18(2):59-65.

8. Cetinkaya Z, Yazar S, Gecici O, Namli MN. AntiToxoplasma gondii antibodies in patients with schizophrenia preliminary findings in a Turkish sample. Schizophr Bull 2007; 33(3):789-791.

9. Özcel MA, Özbel Y, Ak M. Özcel'in tıbbi parazit hastalıkları. Turkey: Parasitology Association, 2007.

10. Ercan F, Demir S. Experienced problems, auditory hallucinations and perceived social support in patients with schizophrenia. Cukurova Med J 2019; 44(1):7-17.

11. Özkul T, Gölgeli A. Development of experimental schizophrenia models and evaluation of schizophrenia symptoms with tests. Mersin Univ J Health Sci 2019; 12(2):351-359.

12. Karakuş G, Tamam L, Zengin M. Hyperprolactinemia and bone metabolism disorders due to antipsychotics in patients with schizophrenia. Anatol J Psychiatry 2009; 10:336-342.

13. Kartalcı Ş, Erbay LG, Zayman EP, Otlu Ö, Karabulut AB, Kartalci G. IL- 4 , TGF- $\beta$, NF- $\kappa$ B and MPO levels in patients with treatment resistant schizophrenia. Turk Psikiyatri Derg 2016; 27(3):170-175.

14. Nimgaonkar VL,. Yolken RH. Neurotropic infectious agents and cognitive impairment in schizophrenia. Schizophr Bull 2012; 38(6):1135-1136.

15. Yolken RH, Torrey EF. Are some cases of psychosis caused by microbial agents? A review of the evidence. Mol Psychiatry 2008; 13(5):470-479.

16. Witting PA. Learning capacity and memory of normal and Toxoplasma-infected laboratory rats and mice. $\mathrm{Z}$ Parasitenkd 1979; 61(1):29-51.

17. Ayaz E, Türkoglu SA, Orallar H. Toxoplasma gondii and epilepsy. Turkiye Parazitol Derg 2016; 40(2):90.

18. Kramer W. Frontiers of neurological diagnosis in acquired toxoplasmosis. Psychiatr Neurol Neurochir 1966; 69(1):43.

19. Minto A, Roberts F. The psychiatric complications of toxoplasmosis. Lancet 1959:1180-1182.

20. Torrey EF, Yolken RH. Could schizophrenia be a viral zoonosis transmitted from house cats? Schizophr Bull 1995; 21(2):167-171.

21. Torrey EF, Rawlings R, Yolken RH. The antecedents of psychoses: a case control study of selected risk factors. Schizophr Res 2000; 46(1):17-23.

22. Alvarado-Esquivel C, Urbina-Álvarez JD, EstradaMartínez S, Torres-Castorena A, Molotla-de-León G, Liesenfeld $\mathrm{O}$ et al. Toxoplasma gondii infection and schizophrenia: a case control study in a low Toxoplasma seroprevalence Mexican population. Parasitol Int 2011; 60(2):151-155.

23. Alipour A, Shojaee S, Mohebali M, Tehranidoost M, Masoleh F A, Keshavarz H. Toxoplasma infection in schizophrenia patients: a comparative study with control group. Iran J Parasitol 2011. 6(2):31.

24. Emelia O, Amal RN, Ruzanna ZZ, Shahida H, Azzubair Z, Tan KS, et al. Seroprevalence of anti-Toxoplasma gondii IgG antibody in patients with schizophrenia. Trop Biomed 2012; 29(1):151-159.

25. Khademvatan S, Khajeddin N, Izadi S, Yousefi E. Investigation of anti-Toxocara and anti-Toxoplasma antibodies in patients with schizophrenia disorder. Schizophr Res Treatment 2014;230-349.

26. Ladee GA. Diagnostic problems in psychiatry with regard to acquired toxoplasmosis. Psychiatr Neurol Neurochir 1966; 69(1):65-82.

27. Yuksel P, Alpay N, Babur C, Bayar R, Saribas S, Karakose AR et al. The role of latent toxoplasmosis in the aetiopathogenesis of schizophrenia the risk factor or an indication of a contact with cat? Folia Parasitol (Praha) 2010; 57(2):121-128.

28. Cevizci S, Celik M, Akcali A, Oyekcin DG, Sahin OO, Bakar C. Seroprevalence of anti-Toxoplasma gondii 
and anti-Borrelia species antibodies in patients with schizophrenia: a case-control study from western Turkey. World J Biol Psychiatry 2015; 16(4):230-236.

29. Tamer GS, Dundar D, Yalug I, Caliskan S, Yazar S, Aker A. The schizophrenia and Toxoplasma gondii connection: infectious, immune or both? Adv Ther 2008; 25(7):703709.

30. Tanyüksel M, Uzun Ö, Araz E, Koruc Ö. Babür C. Possible role of toxoplasmosis in patients with first-episode schizophrenia. Turk J Med Sci 2010; 40(3):399-404.

31. Dogruman-Al F, Aslan S, Yalcin S, Kustimur S, Turk S. A possible relationship between Toxoplasma gondii and schizophrenia: a seroprevalence study. Int J Psychiatry Clin Pract 2009; 13(1):82-87.

32. Arling TA, Yolken RH, Lapidus $M$, Langenberg $P$, Dickerson FB, Zimmerman SA, et al. Toxoplasma gondii antibody titers and history of suicide attempts in patients with recurrent mood disorders. J Nerv Ment Dis 2009; 197(12):905-908.

33. Yagmur F, Yazar S, Temel HO, Cavusoglu M. May Toxoplasma gondii increase suicide attemptpreliminary results in Turkish subjects? Forensic Sci Int 2010; 199(1-3):15-17.

34. Torrey EF, Bartko JJ, Lun ZR, Yolken RH. Antibodies to Toxoplasma gondii in patients with schizophrenia: a meta-analysis. Schizophr Bull 2007; 33(3):729736.

35. Mortensen PB, Nørgaard-Pedersen B, Waltoft BL, Sørensen TL, Hougaard D, Yolken RH. Early infections of Toxoplasma gondii and the later development of schizophrenia. Schizophr Bull 2007; 33(3):741-744. 